\title{
Exact quantum numbers of collapsed and non-collapsed 2-string solutions in the Heisenberg spin chain
}

\author{
Tetsuo Deguchi and Pulak Ranjan Giri \\ Department of Physics, Natural Science Division, Faculty of Core Research, \\ Ochanomizu University \\ 2-1-1 Ohtsuka, Bunkyo-ku, Tokyo 112-8610, Japan \\ E-mail: deguchi@phys.ocha.ac.jp
}

\begin{abstract}
Every solution of the Bethe-ansatz equations (BAE) is characterized by a set of quantum numbers, by which we can evaluate it numerically. However, no general rule is known how to give quantum numbers for the physical solutions of BAE. For the spin- $1 / 2$ XXX chain we rigorously derive all the quantum numbers for the complete set of the Bethe-ansatz eigenvectors in the two downspin sector with any chain length $N$. Here we obtain them both for real and complex solutions. Consequently, we prove the completeness of the Bethe ansatz and give an exact expression for the number of real solutions which correspond to collapsed bound-state solutions (i.e., 2-string solutions) in the sector: $2\left[(N-1) / 2-(N / \pi) \tan ^{-1}(\sqrt{N-1})\right]$ in terms of Gauss' symbol. Moreover, we prove in the sector the scheme conjectured by Takahashi for solving BAE systematically. We also suggest that by applying the present method we can derive the quantum numbers for the spin- $1 / 2 \mathrm{XXZ}$ chain.
\end{abstract}




\section{Introduction}

Exact solutions of the one-dimensional Heisenberg model, i.e. the spin- $1 / 2 \mathrm{XXX}$ chain, were derived by Bethe in 1930s [1] with a systematic method by assuming the form of wavefunctions. We call it the coordinate Bethe ansatz 2]. After Baxter solved the XYZ spin chain, i.e. the eight-vertex model, by introducing and solving the Yang-Baxter equation [3, powerful algebraic methods such as the algebraic Bethe ansatz have been developed [4, 5, 6, 7, 8, where the Yang-Baxter equation leads to algebraic relations such as the commutation relations among the operator elements of the monodromy matrix. However, it is still not trivial whether the eigenvectors constructed by the Bethe-ansatz methods are complete 1, 9. In the Bethe ansatz each Bethe-ansatz eigenvector corresponds to a solution of the Bethe-ansatz equations (BAE). A useful but conjectured scheme was formulated to evaluate all physical solutions of BAE [1, 10, 11. We call it the string hypothesis. In order to confirm it all the solutions are numerically evaluated for the $N$-sited XXX spin chains with $N=10,12$ and 14 [12, 13, 14, (see also [15]).

According to the string hypothesis any solution to BAE is given by either a real number or a sequence of complex numbers, depending on the quantum numbers [10]. We call such a sequence of complex numbers a string. However, it has been shown by Essler, Korepin and Schoutens that some solutions with two down-spins which should be complex in the string hypothesis are indeed real if the system is larger than some critical size [16. We call it the collapse of a potentially complex or bound-state solution to a real solution. The collapse of such a supposed to be complex solution in the string hypothesis to a real solution was observed numerically also in other sectors [12. Thus, in order to study the completeness of the Bethe ansatz, it is fundamental to show whether the number of such new real solutions that appear after some complex solutions collapse corresponds to the number of the missing complex solutions.

It is known that every solution of BAE is characterized by a set of quantum numbers. If the quantum numbers of a complex solution are known, we can numerically evaluate its deviations from the ideal form of strings even though they are very small [14. However, no general rule is known how to give quantum numbers for the physical solutions of BAE. It is thus quite important to determine the quantum number of any physical solution of BAE exactly.

In the present paper we rigorously derive all the quantum numbers for a complete set of the Bethe-ansatz eigenvectors in the sector of two down-spins in the spin- $1 / 2$ XXX chain of any chain length $N$. We first recall that any complex solution with two down-spins is expressed in terms of two real parameters [17. We solve BAE with respect to one of the two parameters and introduce functions which give quantum numbers as their special values. We show that they are monotonic and give the upper and lower bounds to them analytically. We thus obtain the quantum numbers both for real and complex solutions in the sector without making any assumption. Consequently, we prove the completeness of the Bethe-ansatz in the two down-spin sector for any system size $N$ analytically via the Bethe-ansatz equations. Furthermore, we derive an exact expression for the number of missing complex solutions in the sector and that of new real solutions generated after the collapse of complex solutions occurs. We observe that they are equal to each other in the sector for any chain length $N$ : $2\left[(N-1) / 2-(N / \pi) \tan ^{-1}(\sqrt{N-1})\right]$ in terms of Gauss' symbol. Here we remark that the formula has also been obtained independently by Caux [18. Furthermore, we prove the scheme for solving all complex solutions conjectured by Takahashi rigorously 
in the two down-spin sector. In particular, we derive the relation of the Takahashi quantum numbers to the Bethe quantum numbers, which we shall define, shortly in Introduction. Here we make an only one assumption in the paper that the deviations of complex solutions from the complete form (i.e. the string deviations) are very small when we define the Takahashi quantum number. However, the relation between the Bethe and Takahashi quantum numbers is valid even when the deviations are not very small. Based on Ref. 114 we suggest that the result could be related to the characterization of solutions of BAE with rigged configurations [15, 19, 20, 21, 22. Moreover, we confirm some features of complex solutions analytically such as singular string solutions [23, 24, 25, 26, 27, 28, 29, 30, 31] and the large- $N$ behavior of complex solutions [32, 33, 34, 35]. Finally, we suggest that we can derive the quantum numbers of complex solutions with two down-spins for the spin-1/2 XXZ chain, for instance, in the gapful antiferromagnetic regime, by applying the method in the present paper. There are earlier studies on the string solutions of the XXZ spin chain [36, 37, 38, 39.

Let us introduce the Bethe-ansatz eqautions for the XXX spin chain. In the $M$ down-spin sector they are given for $M$ rapidities $\lambda_{1}, \lambda_{2}, \ldots, \lambda_{M}$ in the multiplicative form as follows.

$$
\left(\frac{\lambda_{\alpha}+i / 2}{\lambda_{\alpha}-i / 2}\right)^{N}=\prod_{\beta=1 ; \beta \neq \alpha}^{M} \frac{\lambda_{\alpha}-\lambda_{\beta}+i}{\lambda_{\alpha}-\lambda_{\beta}-i}, \quad \text { for } \alpha=1,2, \ldots, M .
$$

By taking the logarithm of the both hand sides of (1.1) and expressing the logarithmic function of a complex argument in terms of the arctangent function as shown in eq. (A.1) of Appendix A we have

$$
\begin{gathered}
2 \tan ^{-1}\left(2 \lambda_{\alpha}\right)=\frac{2 \pi}{N} J_{\alpha}+\frac{1}{N} \sum_{\beta=1}^{M} 2 \tan ^{-1}\left(\lambda_{\alpha}-\lambda_{\beta}\right), \\
\text { for } \alpha=1,2, \ldots, M .
\end{gathered}
$$

Here we call $J_{\alpha}$ the Bethe quantum numbers [12. They are given by integers or half-integers according to the condition

$$
J_{\alpha}=\frac{1}{2}(N-M+1) \quad(\bmod 1) .
$$

In the paper we take the branch of the arctangent function: $-\pi / 2<\tan ^{-1} x<\pi / 2$ for any $x \in \boldsymbol{R}$, and hence we do not assume any additional integral multiple of $2 \pi$ in (1.2).

In the string hypothesis we assume that any solution to the Bethe-ansatz equations (1.1) is given by a sequence of $n$ complex numbers which are different from each other by integral multiples of an imaginary number such as $i$, at least approximately:

$$
\lambda_{\alpha k}^{n}=x_{\alpha}^{n}+\frac{i}{2}(n+1-2 k)+\Delta_{\alpha k}^{n} \quad \text { for } k=1,2, \ldots, n .
$$

We call the set of rapidities of the form (1.4) an $n$-string. We call $x_{\alpha}^{n}$ the center of the $n$-string and $\Delta_{\alpha k}^{n}$ the deviations from the complete $n$-string. Here, suffix $\alpha$ being the index to distinguish all the $M_{n}$ strings of the same length $\Delta_{\alpha k}^{n}$ are the string deviations. We remark that a real solution is given by 1-strings, which are given by putting $n=1$ in (1.4). In the string hypothesis we assume that the absolute values of 
the deviations are very small: $\left|\Delta_{\alpha k}^{n}\right| \ll 1$. In the limit that these deviations vanish, $\Delta_{\alpha k}^{n} \rightarrow 0$, the Bethe-ansatz equations reduce to the convenient form

$$
\begin{aligned}
\tan ^{-1}\left(\frac{2 \lambda_{\alpha}^{n}}{n}\right)= & \pi \frac{I_{\alpha}^{n}}{N}+\frac{1}{N} \sum_{k=1}^{N_{s}} \sum_{\beta=1}^{M_{k}} \Theta_{n k}\left(\lambda_{\alpha}^{n}-\lambda_{\beta}^{k}\right) \\
\Theta_{j k}(\lambda)= & \left(1-\delta_{n k}\right) \tan ^{-1}\left(\frac{2 \lambda}{|n-k|}\right)+\tan ^{-1}\left(\frac{2 \lambda}{|n-k|+2}\right)+\cdots \\
& +\cdots+2 \tan ^{-1}\left(\frac{2 \lambda}{n+k-2}\right)+\tan ^{-1}\left(\frac{2 \lambda}{n+k}\right), \quad(1.5)
\end{aligned}
$$

where $M$ down-spins are partitioned into $M_{k} k$-stings with the length of the largest string being $N_{s}$ such that $\sum_{k}^{N_{s}} k M_{k}=M$. The reduced equations (1.5) are called the Takahashi (or the Bethe-Takahashi) equations. We now obtain the strictly nonrepetitive quantum numbers $I_{\alpha}^{j}$, known as the Takahashi quantum numbers, from eq. (1.5), which have the following bounds

$$
\left|I_{\alpha}^{j}\right| \leq \frac{1}{2}\left(N-1-\sum_{k=1}\left[2 \min (j, k)-\delta_{j, k}\right] M_{k}\right) .
$$

We recall that it has not been clearly shown previously how to derive all the possible Bethe quantum numbers for the Bethe-ansatz equations (1.2) with $M$ downspins even for the $M=2$ case. Furthermore, we observe in some examples that the same numbers appear in the Bethe quantum numbers for different rapidities, i.e. the Bethe quantum numbers are repetitive. However, if we assume the string hypothesis then the Takahashi quantum numbers (or the Bethe-Takahashi quantum numbers [12]) are not repetitive, and hence are indeed useful for not only classifying but also evaluating solutions to the Bethe-ansatz equations numerically.

The contents of the paper consist of the following. In section 2 we derive rigorously the Bethe quantum numbers for 2-string solutions in the two down-spin sector. In section 3 we derive rigorously the Bethe quantum numbers for 1-string solutions in the two down-spin sector. Here we extend the parameters of complex solutions to those of a pair of real solutions. In section 4 we show some features of 2 -string solutions with two down-spins. In section 5 we show analytically the relation between the Bethe quantum numbers and the Takahashi quantum numbers. We show that the Takahashi quantum numbers are distinct, while the Bethe quantum numbers may be repetitive although they are distinct as pairs. In section 6 we give some remarks on how far we have shown the completeness of the Bethe ansatz, and on suggestions for further studies.

\section{Bethe quantum numbers of the complex solutions with two down-spins}

\subsection{Assumed form of a 2-string solution}

Le us recall that every physical solution is self-conjugate under complex conjugation in the XXX spin chain [17]. We therefore assume the following form of a 2-string solution to the Bethe-ansatz equations (1.2) in the two down-spin sector $(M=2)$ :

$$
\begin{aligned}
& \lambda_{1}=x+\frac{i}{2}(1+2 \delta), \\
& \lambda_{2}=x-\frac{i}{2}(1+2 \delta) .
\end{aligned}
$$


Here we assume that both the string center $x$ and the string deviation $\delta$ are real. Moreover, we assume that $-1 / 2 \leq \delta$ due to the symmetry between $\lambda_{1}$ and $\lambda_{2}$. We shall consider two regimes of $\delta$ : the positive regime with $\delta>0$ and the negative regime satisfying $-1 / 2<\delta<0$. The Bethe-ansatz equations in the logarithmic form for a 2-string with $M=2$ are explicitly given by

$$
\begin{aligned}
& 2 \tan ^{-1}(2 x+i(1+2 \delta))=\frac{2 \pi}{N} J_{1}+\frac{1}{N} 2 \tan ^{-1}(i(1+2 \delta)), \\
& 2 \tan ^{-1}(2 x-i(1+2 \delta))=\frac{2 \pi}{N} J_{2}+\frac{1}{N} 2 \tan ^{-1}(-i(1+2 \delta)) .
\end{aligned}
$$

Let us now solve the Bethe-ansatz equations (2.2) and (2.3) analytically without making any approximation. We introduce the step function $H(x)$ by

$$
H(x)= \begin{cases}1 & \text { for } x>0 \\ 0 & \text { otherwise }\end{cases}
$$

We also define $\operatorname{sign}$ functions $\operatorname{sgn}\left(x_{ \pm}\right)$by $\operatorname{sgn}\left(x_{+}\right)=1-2 H(-x)$ and $\operatorname{sgn}\left(x_{-}\right)=$ $2 H(x)-1$, respectively. By applying the expression of the arctangent function with a complex argument given in eq. (A.4) we calculate the left-hand side of (2.2) as follows.

$$
\begin{gathered}
2 \tan ^{-1}(2 x+i(1+2 \delta))=\tan ^{-1}\left(\frac{x}{1+\delta}\right)-\tan ^{-1}\left(\frac{x}{\delta}\right) \\
+\pi H(\delta) \operatorname{sgn}\left(x_{+}\right)+\frac{1}{2 i} \log \left(\frac{x^{2}+\delta^{2}}{x^{2}+(1+\delta)^{2}}\right) .
\end{gathered}
$$

Here we have considered $H(-2-2 \delta)=0$ since $\delta>-1 / 2$. Moreover, in the paper we assume the branch of logarithmic function $\log z$ satisfying $|\Im \log z| \leq \pi$, which is given by setting $n=0$ in A.1 , if we do not specify it otherwise.

Let us define counting functions $Z_{ \pm}(x, \delta)$, which are functions of the two variables, string center $x$ and string deviation $\delta$, by

$$
\begin{aligned}
2 \pi Z_{ \pm}(x, \delta)= & \pi H(\delta)\left(\operatorname{sgn}\left(x_{ \pm}\right)-( \pm 1) \frac{1}{N}\right) \\
& +\tan ^{-1}\left(\frac{x}{1+\delta}\right)-\tan ^{-1}\left(\frac{x}{\delta}\right) .
\end{aligned}
$$

Through (2.5) we show that the first BAE (2.2) holds if the following equations are satisfied:

$$
\begin{aligned}
& \frac{2 \pi}{N} J_{1}=2 \pi Z_{+}(x, \delta), \\
& \log \left(\frac{x^{2}+\delta^{2}}{x^{2}+(1+\delta)^{2}}\right)=\frac{1}{N} \log \left(\frac{\delta^{2}}{(1+\delta)^{2}}\right) .
\end{aligned}
$$

Applying formula (2.5) to the second BAE (2.3), where we only replace $i$ with $-i$, we show that it holds if eq. (2.8) and the following equation are satisfied:

$$
\frac{2 \pi}{N} J_{2}=2 \pi Z_{-}(x, \delta) \text {. }
$$

Thus, BAEs (2.2) and (2.3) are derived from the three equations (2.7), (2.8) and (2.9), and if we take the branch of $\log z$ with $|\operatorname{Im} \log z| \leq \pi$, they are equivalent to the BAEs.

For the 2-string solutions the Bethe quantum numbers $J_{1}$ and $J_{2}$ are not independent of each other. By taking the difference of (2.7) and (2.9) for $x \neq 0$ we have the relation between them.

$$
J_{2}=J_{1}+H(\delta) \text {. }
$$


We now express the square of string center $x^{2}$ as a function of string deviation $\delta$. By taking the exponential of (2.8) we have

$$
\frac{x^{2}+\delta^{2}}{x^{2}+(1+\delta)^{2}}=\left(\frac{\delta^{2}}{(1+\delta)^{2}}\right)^{1 / N}
$$

We therefore obtain the following expression of $x^{2}$ as a function of $\delta$ :

$$
x^{2}=(1+\delta)^{2} \frac{\left(\delta^{2} /(1+\delta)^{2}\right)^{1 / N}-\delta^{2} /(1+\delta)^{2}}{1-\left(\delta^{2} /(1+\delta)^{2}\right)^{1 / N}} .
$$

\subsection{Cases of the string deviation being real}

In order to parametrize string deviation $\delta$ we introduce variable $\beta$ by

$$
\delta=-\frac{1}{2}+\frac{\beta}{2} \quad(\beta \geq 0) .
$$

For positive $\delta(\delta>0)$ we have $\beta>1$, while for negative $\delta(-1 / 2<\delta<0)$ we have $0<\beta<1$.

We define an important variable $w$ by

$$
w=\frac{\delta^{2}}{(1+\delta)^{2}} .
$$

In terms of $\beta$ we have $w=(\beta-1)^{2} /(1+\beta)^{2}$. For $\delta \geq 0$, variable $w$ increases from 0 to 1 as $\beta$ increases from 1 to $\infty$. For $-1 / 2<\delta \leq 0$, variable $w$ increases from 0 to 1 as $\beta$ decreases from 1 to 0 . We shall show later that the 2 -string solution collapses when $\beta$ approaches $0(\delta=-1 / 2)$.

It follows from (2.12) that ratios $x^{2} /(1+\delta)^{2}$ and $x^{2} / \delta^{2}$ are expressed as functions of single variable $w$.

$$
\frac{x^{2}}{(1+\delta)^{2}}=\frac{w^{1 / N}-w}{1-w^{1 / N}}, \quad \frac{x^{2}}{\delta^{2}}=\frac{w^{1 / N-1}-1}{1-w^{1 / N}} .
$$

We now consider four cases with respect to string deviation $\delta$ and string center $x$ : (i) $\delta>0$ and $x>0$; (ii) $\delta>0$ and $x<0$; (iii) $\delta<0$ and $x>0$; (iv) $\delta<0$ and $x<0$. By taking the square roots of $x^{2} /(1+\delta)^{2}$ and $x^{2} / \delta^{2}$ ratios $x /(1+\delta)$ and $x / \delta$ are expressed in terms of $\epsilon_{1}= \pm 1$ and $\epsilon_{2}= \pm 1$ as

$$
\frac{x}{1+\delta}(w)=\epsilon_{1} \sqrt{\frac{w^{1 / N}-w}{1-w^{1 / N}}}, \quad \frac{x}{\delta}(w)=\epsilon_{2} \sqrt{\frac{w^{1 / N-1}-1}{1-w^{1 / N}}} .
$$

In terms of signs $\epsilon_{1}$ and $\epsilon_{2}$ in (2.16) we specify cases (i), (ii), (iii) and (iv) with $\left(\epsilon_{1}, \epsilon_{2}\right)=(+,+),(-,-),(+,-)$ and $(-,+)$, respectively. Consequently, we have $\delta /(1+\delta)=\epsilon_{1} \epsilon_{2} w^{1 / 2}$ and we can express $\delta$ as a function of $w$.

$$
\delta=\frac{1}{1-\epsilon_{1} \epsilon_{2} w^{1 / 2}}-1
$$

We define $\Theta(w)$ by

$$
\Theta(w)=\tan ^{-1}\left(\frac{x}{1+\delta}(w)\right)-\tan ^{-1}\left(\frac{x}{\delta}(w)\right) .
$$

Thus, by solving the constraint (2.8) in terms of $w$, we express the counting functions $Z_{ \pm}(x, \delta)$ as $Z_{ \pm}(x(w), \delta(w))$ as functions of $w$ for $0 \leq w \leq 1$.

$$
2 \pi Z_{ \pm}(x(w), \delta(w))=\Theta(w)+\pi H(\delta(w))\left(\operatorname{sgn}\left(x(w)+0_{ \pm}\right)-( \pm 1) \frac{1}{N}\right)
$$


Through direct calculation we can show

$$
\frac{d \Theta}{d w}=\frac{\epsilon_{1}(1-w)\left(w^{1 / N-1 / 2}-1\right)+\epsilon_{2} N\left(1-w^{1 / 2}\right)\left(1-w^{1 / N}\right)}{2 N(1-w) \sqrt{\left(1-w^{1 / N}\right)\left(w^{1 / N-1}-1\right)}} .
$$

It is easy to show that

$$
(1-w)\left(w^{1 / N-1 / 2}-1\right)+N\left(1-w^{1 / 2}\right)\left(1-w^{1 / N}\right)>0 \quad \text { for } 0<w<1 .(2.21)
$$

Furthermore, we can show the following lemma (the proof is given in Appendix B).

Lemma 2.1 For $0<w<1$ we have

$$
(1-w)\left(1+w^{1 / N-1 / 2}\right)-N\left(1+w^{1 / 2}\right)\left(1-w^{1 / N}\right)>0 .
$$

It follows from (2.20), (2.21) and (2.22) that we have either $d \Theta / d w>0$ or $d \Theta / d w<0$ in each of the four cases from (i) to (iv). If $d \Theta / d w>0$, the lower bound (or the minimum) and the upper bound of function $\Theta(w)$ are given by the limiting value of $\Theta(w=0)$ when we send $w$ to zero with $w>0$ and that of $\Theta(w=1)$ when we send $w$ to 1 , respectively. If $d \Theta / d w<0$, the upper bound and the lower bound (or the minimum) of function $\Theta(w)$ are given by the limiting value of $\Theta(w=0)$ when we send $w$ to zero with $w>0$ and that of $\Theta(w=1)$ when we send $w$ to 1 , respectively.

By making use of the positivity (or negativity) of the derivative of the counting function $Z_{ \pm}(x(w), \delta(w))$ with respect to $w$ we have the following results.

Case (i): $\delta>0$ (i.e., $1<\beta$ ) and $x>0$

$$
\frac{N}{4}-\frac{1}{2} \leq J_{1}<\frac{N}{2}-\frac{1}{2} .
$$

Case (ii): $\delta>0$ (i.e., $1<\beta$ ) and $x<0$

$$
-\frac{N}{2}-\frac{1}{2}<J_{1} \leq-\frac{N}{4}-\frac{1}{2} .
$$

Case (iii): $\delta<0$ (i.e., $0<\beta<1$ ) and $x>0$.

$$
\frac{N}{4} \leq J_{1}<\frac{N}{\pi} \tan ^{-1}(\sqrt{N-1}) .
$$

Case (iv): $\delta<0$ (i.e., $0<\beta<1) x<0$

$$
-\frac{N}{\pi} \tan ^{-1}(\sqrt{N-1}) \leq J_{1}<-\frac{N}{4} .
$$

Here we recall that the quantum numbers $J_{\alpha}$ are given by integers or half-odd integers, which satisfy the condition (1.3).

\subsection{Exact number of missing 2-string solutions}

We now derive analytically an exact expression for the number of missing 2-string solutions in the XXX spin chain of $N$ sites, $N_{\text {missing }}$. Here we remark that it is easy to show the following inequality:

$$
\frac{N}{\pi} \tan ^{-1}(\sqrt{N-1})<\frac{N-1}{2} \text { for } N>2 .
$$

We also remark that when $J<(N-1) / 2$ the largest Bethe quantum number $J$ satisfying the condition (1.3) is given by $J=(N-3) / 2$ both for even $N$ and odd $N$.

In the case (iii) when $\delta<0$ and $x>0$ the Bethe quantum number $J_{1}$ has the upper bound $(N / \pi) \tan ^{-1}(\sqrt{N-1})$. It corresponds to the case of $\beta=0$ (i.e. $\delta=-1 / 2$ ), 
which does not happen for any finite-size system. Therefore, if the upper bound is equal to the largest possible quantum number $(N-3) / 2$, the 2 -string solution does not exist. Thus, the number of missing 2 -string solutions in the case (iii) is given by $\left[(N-3) / 2-(N / \pi) \tan ^{-1}(\sqrt{N-1})+1\right]$. Here, the symbol $[x]$ denotes Gauss' symbol, which expresses the largest integer that is less than or equal to $x$.

By arguing the case (iv) similarly, we obtain an exact estimate $N_{\text {missing }}$ for the number of missing 2-string solutions in the XXX spin chain with $N$ sites as follows.

$$
N_{\text {missing }}=2\left[\frac{N-1}{2}-\frac{N}{\pi} \tan ^{-1}(\sqrt{N-1})\right] .
$$

Here we recall that $[x]$ denotes Gauss' symbol.

\subsection{Critical lattice size for the collapse of a 2-string solution}

We now derive the critical lattice size $N$ for which the number of missing 2-string solutions becomes larger or equal to 1 . We define $N_{c}$ as follows. For $N<N_{c}$ we have $N_{\text {missing }}=0$, while for $N \geq N_{c}$ we have $N_{\text {missing }} \geq 1$, i.e. the number of missing 2-string solutions becomes nonzero.

It was first shown by Essler, Korepin and Schoutens that the critical lattice size $N_{c}$ is given by $N_{c}=22$ [16]. We confirm it by making use of (2.28) as follows. For $N=22$ we have the largest Bethe quantum number $J_{1}=(N-3) / 2=19 / 2=9.5$. On the other hand, by putting $N=22$ in (2.28) we have

$$
\frac{22}{\pi} \tan ^{-1}(\sqrt{22-1})=9.49545,
$$

which is slightly smaller than 9.5. Moreover, it is straightforward to show that $N_{\text {missing }}=0$ for $N<22$. Therefore, the critical lattice size $N_{c}$ is given by $N_{c}=22$.

\section{Bethe quantum numbers of the real solutions with two-down spins}

\subsection{Real solutions corresponding collapsed 2-string solutions}

Let us extend the string deviation $\delta$ to complex values by substituting $\beta=i \gamma$ with $\gamma \geq 0$ in (2.13) as follows.

$$
\delta=-\frac{1}{2}+\frac{i}{2} \gamma .
$$

We now search for real solutions consisting of two rapidities of the following form:

$$
\begin{aligned}
& \lambda_{1}=x+i(1+2 \delta) / 2=x-\gamma, \\
& \lambda_{2}=x-i(1+2 \delta) / 2=x+\gamma .
\end{aligned}
$$

We call parameter $x$ the center and $\gamma$ the deviation of a real solution with two-down spins, $\lambda_{1}$ and $\lambda_{2}$.

By expressing the arctangent functions in terms of the logarithmic functions through (A.3) with $b=0$ we first show

$$
\begin{aligned}
& 2 \tan ^{-1}(2 x+i(1+2 \delta))=2 \tan ^{-1}(2 x-\gamma) \\
& =\frac{1}{i} \log \left(\frac{1+i(2 x-\gamma)}{1-i(2 x-\gamma)}\right) \\
& =\frac{1}{2 i} \log \left(\frac{1+i(2 x-\gamma)}{1-i(2 x-\gamma)}\right)+\frac{1}{2 i} \log \left(\frac{1+i(2 x+\gamma)}{1-i(2 x+\gamma)}\right)
\end{aligned}
$$


Exact quantum numbers of collapsed 2-string solutions

$$
\begin{aligned}
& +\frac{1}{2 i} \log \left(\frac{1+i(2 x-\gamma)}{1-i(2 x-\gamma)}\right)-\frac{1}{2 i} \log \left(\frac{1+i(2 x+\gamma)}{1-i(2 x+\gamma)}\right) \\
& =\tan ^{-1}(2 x+\gamma)+\tan ^{-1}(2 x-\gamma)+\frac{1}{2 i} \log \left(\frac{(1-i \gamma)^{2}+4 x^{2}}{(1+i \gamma)^{2}+4 x^{2}}\right) .
\end{aligned}
$$

By making use of (3.3) we have

$$
\begin{aligned}
& 2 \tan ^{-1}(2 x+i(1+2 \delta))-\frac{1}{N} 2 \tan ^{-1}(i(1+2 \delta)) \\
& =\tan ^{-1}(2 x-\gamma)+\tan ^{-1}(2 x+\gamma) \\
& +\frac{1}{2 i}\left\{\log \left(\frac{(1-i \gamma)^{2}+4 x^{2}}{(1+i \gamma)^{2}+4 x^{2}}\right)-\frac{1}{N} \log \left(\frac{(1-i \gamma)^{2}}{(1+i \gamma)^{2}}\right)\right\} .
\end{aligned}
$$

We now define a counting function $W(x, \gamma)$ by

$$
2 \pi W(x, \gamma)=\tan ^{-1}(2 x-\gamma)+\tan ^{-1}(2 x+\gamma) .
$$

The Bethe-ansatz equations (2.2) and (2.3) with the complex-valued string deviation $\delta$ (3.1) are expressed as follows.

$$
\begin{aligned}
& \frac{2 \pi}{N} J_{1}=2 \pi W(x, \gamma) \\
& \quad-\frac{1}{2 i}\left(\log \left(\frac{(1+i \gamma)^{2}+4 x^{2}}{(1-i \gamma)^{2}+4 x^{2}}\right)-\frac{1}{N} \log \left(\frac{(1+i \gamma)^{2}}{(1-i \gamma)^{2}}\right)\right), \\
& \frac{2 \pi}{N} J_{2}=2 \pi W(x, \gamma) \\
& \quad+\frac{1}{2 i}\left(\log \left(\frac{(1+i \gamma)^{2}+4 x^{2}}{(1-i \gamma)^{2}+4 x^{2}}\right)-\frac{1}{N} \log \left(\frac{(1+i \gamma)^{2}}{(1-i \gamma)^{2}}\right)\right) .
\end{aligned}
$$

Here we remark that when we take the $N$ th root of a complex $\operatorname{argument}$ of $\log z$, we may introduce an $N$ th root of unity with an integer $n$ as follows.

$$
\frac{1}{N} \log \left(\frac{(1+i \gamma)^{2}}{(1-i \gamma)^{2}}\right)=\log \left\{\exp \left(\frac{2 \pi i n}{N}\right)\left(\frac{(1+i \gamma)^{2}}{(1-i \gamma)^{2}}\right)^{1 / N}\right\} .
$$

Suggested by the above remark, we now assume the following relation for $m=$ $0,1, \ldots, N-1$ :

$$
\frac{(1+i \gamma)^{2}+4 x^{2}}{(1-i \gamma)^{2}+4 x^{2}}=\exp \left(\frac{2 \pi i m}{N}\right) \times\left(\frac{(1+i \gamma)^{2}}{(1-i \gamma)^{2}}\right)^{1 / N} .
$$

It follows that the Bethe-ansatz equations (3.6) and (3.7) are derived from eq. (3.9) and the following equations for $m=0,1, \ldots, N-1$ :

$$
\begin{aligned}
& \frac{2 \pi}{N} J_{1}=2 \pi W(x, \gamma), \\
& J_{1}=J_{2}-m .
\end{aligned}
$$

We now solve constraint (3.9) on center $x$ and express $x$ as a function of $\gamma$ with $\gamma \geq 0$. Let us introduce variable $\varphi$ by $\varphi=\tan ^{-1} \gamma$. It satisfies $0 \leq \varphi<\pi / 2$. From (3.9) we express the square of center $x^{2}$ as a function of $\varphi$ as follows.

$$
x^{2}(\varphi)=\frac{1}{4 \cos ^{2} \varphi} \frac{\sin (2 \varphi-(2 \varphi+m \pi) / N)}{\sin ((2 \varphi+m \pi) / N)} \quad(m=0,1, \ldots, N-1) .
$$

In subsections 3.1 and 3.2 we consider only the case of $m=0$ and show that the number of real solutions with $m=0$ is equal to the number of missing complex 
solutions. However, the solutions for other values of $m$ correspond to the standard real solutions with two 1-strings, as we shall see in section 3.3. We thus derive all the quantum numbers in the two down-spin sector including both real and complex solutions, simply by extending the string deviation $\delta$ into pure imaginary values.

If we put $m=0$ in (3.12), it is clear that the right-hand side is positive for the whole range of $\varphi$ : $0<\varphi<\pi / 2$. Thus, by introducing the sign factor: $\epsilon_{3}= \pm 1$, the center $x(\varphi)$ as a function of $\varphi$ is given by

$$
x(\varphi)=\frac{\epsilon_{3}}{2 \cos \varphi} \sqrt{\frac{\sin (2 \varphi(1-1 / N))}{\sin (2 \varphi / N)}} \quad(0<\varphi<\pi / 2) .
$$

We shall consider two cases: (v) $x>0\left(\epsilon_{3}=+1\right)$ and (vi) $x<0\left(\epsilon_{3}=-1\right)$. Through direct calculation we derive

$$
\frac{d}{d \varphi} x^{2}=\frac{\sin (2 \varphi / N) \cos (\varphi-2 \varphi / N)-(1 / N) \sin (2 \varphi) \cos \varphi}{2 \cos ^{3} \varphi \sin ^{2}(2 \varphi / N)} .
$$

By proving explicitly that the enumerator of (3.14) is positive for $0<\varphi<\pi / 2$ we show that the square of center, $x(\varphi)^{2}$, is monotonically increasing:

$$
\frac{d}{d \varphi} x^{2}>0 \text { for } 0<\varphi<\pi / 2 .
$$

In order to show the monotonicity of the square of center $x(\varphi)^{2}$ in shown (3.15) we make use of an inequality: $\cos (\varphi-2 \varphi / N)>\cos \varphi$ for $0<\varphi<\pi / 2$ and the following lemma:

Lemma 3.1 For $\alpha$ satisfying $0<\alpha<1$ we have

$$
\sin \alpha x>\alpha \sin x \quad(0<x<\pi) .
$$

We show lemma 3.1 by taking the derivative of (3.16) with respect to center $x$.

It thus follows from (3.15) that we have the minimum value of the square of center $x(\varphi)^{2}$ when we send $\varphi$ to zero.

$$
\lim _{\varphi \rightarrow 0} x(\varphi)^{2}=\frac{1}{4} \frac{1-1 / N}{1 / N}=\frac{N-1}{4} .
$$

The value of $x(\varphi)^{2}$ becomes infinite as we send $\varphi$ to $\pi / 2$.

We define $\kappa(\varphi)$ by $\kappa(\varphi)=2 \pi W(x(\varphi), \gamma(\varphi))$

$$
\kappa(\varphi)=\tan ^{-1}(2 x(\varphi)-\gamma(\varphi))+\tan ^{-1}(2 x(\varphi)+\gamma(\varphi)) .
$$

We calculate the derivative of $\kappa(\varphi)$ as

$$
\begin{aligned}
\frac{d \kappa}{d \varphi} & =\frac{4\left(1+4 x^{2}+\gamma^{2}\right) \frac{d x}{d \varphi}-8 x \gamma \frac{d \gamma}{d \varphi}}{\left\{(2 x-\gamma)^{2}+1\right\}\left\{(2 x+\gamma)^{2}+1\right\}} \\
& =\frac{\left(4 x^{2}+1 / \cos ^{2} \varphi\right) \frac{d}{d \varphi} x^{2}-4 x^{2} \frac{\sin \varphi}{\cos ^{3} \varphi}}{x / 2\left\{(2 x-\gamma)^{2}+1\right\}\left\{(2 x+\gamma)^{2}+1\right\}} .
\end{aligned}
$$

We shall show in Appendix $\mathrm{C}$ the following inequality:

$$
\left(4 x^{2}+\frac{1}{\cos ^{2} \varphi}\right) \frac{d}{d \varphi} x^{2}>4 x^{2} \frac{\sin \varphi}{\cos ^{3} \varphi} \quad(0<\varphi<\pi / 2) .
$$

It follows that for $x>0$ the derivative of $\kappa(\varphi)$ is positive for $0<\varphi<\pi / 2$ while for $x<0$ the derivative of $\kappa(\varphi)$ is negative for $0<\varphi<\pi / 2$. Hence, the minimum and 
the maximum values of $\kappa(\varphi)$ for $x>0$ are given by $\kappa(0)$ and $\kappa(\pi / 2)$, respectively, while the minimum and the maximum values of $\kappa(\varphi)$ for $x<0$ by $\kappa(\pi / 2)$ and $\kappa(0)$, respectively.

We now consider the case of $x>0$. We calculate $\kappa(\pi / 2)$ by sending $\varphi$ to $\pi / 2$ with $\varphi<\pi / 2$. In the limit of sending $\varphi$ to $\pi / 2$ the term $2 x(\varphi)+\gamma(\varphi)$ approaches $\infty$ and hence we have

$$
\lim _{\varphi \rightarrow \pi / 2} \tan ^{-1}(2 x(\varphi)+\gamma(\varphi))=\pi / 2 .
$$

For the term $2 x-\gamma$, by putting $\varphi=\pi / 2-\epsilon$ into it and by expanding it in terms of $\epsilon$ we show

$$
\lim _{\varphi \rightarrow \pi / 2} 2 x(\varphi)-\gamma(\varphi)=\cot \left(\frac{\pi}{N}\right) .
$$

We remark that $\cot x=\tan (\pi / 2-x)$. We therefore have

$$
\begin{aligned}
\lim _{\varphi \rightarrow \pi / 2} \kappa(\varphi) & =\left(\frac{\pi}{2}-\frac{\pi}{N}\right)+\frac{\pi}{2} \\
& =\pi-\frac{\pi}{N} .
\end{aligned}
$$

We calculate $\kappa(0)$ by sending $\varphi$ to 0 with $\varphi>0$.

$$
\lim _{\varphi \rightarrow 0} \kappa(\varphi)=2 \tan ^{-1}(\sqrt{N-1}) .
$$

We thus have the following results.

Case (v) : $x>0$ with $\delta=(-1+i \gamma) / 2$

$$
\frac{N}{\pi} \tan ^{-1}(\sqrt{N-1}) \leq J_{1}<\frac{N}{2}-\frac{1}{2}
$$

Case (vi) : $x<0$ with $\delta=(-1+i \gamma) / 2$

$$
-\frac{N}{2}+\frac{1}{2}<J_{1} \leq-\frac{N}{\pi} \tan ^{-1}(\sqrt{N-1})
$$

Here we assume that if $\varphi=0$ we have a real solution with a pair of the same rapidities.

\subsection{Number of new real solutions corresponds to that of missing 2-string solutions}

In the case (v) when $x>0$ with complex-valued deviation $\delta$, the largest Bethe quantum number $J_{1}$ is given by $(N-3) / 2$ both for even and odd $N$, as shown in subsection 2.3. Here the smallest Bethe quantum number is given by an integer or a half-integer greater than or equal to $(N / \pi) \tan ^{-1}(\sqrt{N-1})$. Therefore, the number of new real solutions in the case (v) is given by $N_{\text {new }}=\left[(N-3) / 2-N / \pi \tan ^{-1}(\sqrt{N-1})+1\right]$.

Similarly, we have in the case (vi) when $x<0$, we have the number of new real solutions $\left[(N-3) / 2-N / \pi \tan ^{-1}(\sqrt{N-1})+1\right]$. In total, we have the same number in the case of (vi).

Combining two cases (v) and (vi) we give the number of new real solutions is given by

$$
N_{\text {new }}=2\left[\frac{N-1}{2}-\frac{N}{\pi} \tan ^{-1}(\sqrt{N-1})\right] .
$$

Thus, the number of missing complex solutions is exactly equal to the number of new real solutions. Moreover, the new real solutions have the same Bethe quantum numbers with those of the missing complex solutions. 


\subsection{Standard real solutions as 2-strings with imaginary deviations}

In the case of $m \neq 0$ such as $m=1,2, \ldots, N-1$ for eq. (3.12) we express $x(\varphi)$ as follows.

$$
x(\varphi)=\frac{\epsilon_{3}}{2 \cos \varphi} \sqrt{\frac{\sin (2 \varphi-(2 \varphi+m \pi) / N)}{\sin ((2 \varphi+m \pi) / N)}} .
$$

Since $x(\varphi)^{2}$ must be non-negative, the range of parameter $\varphi$ is given by

$$
\frac{m \pi}{2(N-1)} \leq \varphi<\frac{\pi}{2} \quad \text { for } \quad m=1,2, \ldots, N-1 .
$$

Here we do not consider the case of $m=N-1$ since there is no range for $\varphi$ satisfying (3.29). We denote the minimum value of $\varphi$ by $\varphi_{\min }: \varphi=m \pi / 2(N-1)$. By taking the derivative of the square of center $x(\varphi)^{2}$ with respect to variable $\varphi$, we have

$$
\begin{aligned}
& \frac{d}{d \varphi} x(\varphi)^{2} \\
& =\frac{\sin ((2 \varphi+m \pi) / N) \cos (\varphi-(2 \varphi+m \pi) / N)-(1 / N) \sin (2 \varphi) \cos \varphi}{2 \cos ^{3} \varphi \sin ^{2}((2 \varphi+m \pi) / N)} .
\end{aligned}
$$

Proving explicitly that the enumerator of (3.30) is positive for $m \pi /(2(N-1)) \leq$ $\varphi<\pi / 2$ in Appendix C, we show that the square of center, $x(\varphi)^{2}$, is monotonically increasing in the region: $\varphi_{\min } \leq \varphi<\pi / 2$ :

$$
\frac{d}{d \varphi} x^{2}>0 \quad \text { for } \quad \frac{m \pi}{2(N-1)}<\varphi<\pi / 2 .
$$

In order to show (3.31) we make use of an inequality:

$$
\cos \left(\varphi-\frac{2 \varphi+m \pi}{N}\right)>\cos \varphi \text { for } \frac{m \pi}{2(N-1)}<\varphi<\frac{\pi}{2} .
$$

and the next lemma.

Lemma 3.2 For any given real number $N$ satisfying $N>1$ we have

$$
\sin \left(\frac{2(\varphi+m \pi)}{N}\right)>\frac{1}{N} \sin 2 \varphi \text { for } \frac{m \pi}{2(N-1)}<\varphi<\frac{\pi}{2} .
$$

We can prove lemma 3.2 by taking the derivative of (3.33) with respect to center $x$.

It follows from (3.32) and (3.33) that we have the following inequality:

$$
\begin{gathered}
\sin \left(\frac{2(\varphi+m \pi)}{N}\right) \cos \left(\varphi-\frac{2 \varphi+m \pi}{N}\right)>\frac{1}{N} \sin 2 \varphi \cos \varphi \\
\text { for } \frac{m \pi}{2(N-1)}<\varphi<\frac{\pi}{2} .
\end{gathered}
$$

It follows that inequality (3.31) holds, and hence the square of center $x(\varphi)^{2}$ is monotonic increasing.

We define function $\kappa(\varphi)$ by (3.18) also for the cases of $m=1,2, \ldots, N-1$. Here we recall that the derivative of $\kappa(\varphi)$ with respect to $\varphi$ is given in eq. (3.19). We shall show in Appendix $\mathrm{D}$ the following inequality for $m=1,2, \ldots, N-2$ :

$$
\left(4 x^{2}+\frac{1}{\cos ^{2} \varphi}\right) \frac{d}{d \varphi} x^{2}>4 x^{2} \frac{\sin \varphi}{\cos ^{3} \varphi} \text { for } \frac{m \pi}{2(N-1)} \leq \varphi<\frac{\pi}{2}
$$


It therefore follows that $\kappa(\varphi)$ is monotonically increasing function in the interval with $\pi /(2(N-1))<\varphi<\pi / 2$.

We consider case (vii) when $x>0$ and case (viii) when $x<0$. For $x>0$ we can show that the derivative of $\kappa(\varphi)$ is positive for $\varphi_{\min }<\varphi<\pi / 2$. Hence, the minimum and maximum of $\kappa(\varphi)$ are given by $\kappa\left(\varphi_{\min }\right)$ and $\kappa(\pi / 2)$, respectively. We calculate $\kappa(\pi / 2)$ by sending $\varphi$ to $\pi / 2$ with $\varphi<\pi / 2$. In the limit of sending $\varphi$ to $\pi / 2$ the term $2 x(\varphi)+\gamma(\varphi)$ approaches infinity, and hence we have

$$
\lim _{\varphi \rightarrow \pi / 2} \tan ^{-1}(2 x(\varphi)+\gamma(\varphi))=\pi / 2,
$$

For $2 x-\gamma$, by putting $\varphi=\pi / 2-\epsilon$ and expanding (3.28) with respect to $\epsilon$, we show

$$
\lim _{\varphi \rightarrow \pi / 2} 2 x(\varphi)-\gamma(\varphi)=\cot \left(\frac{(m+1) \pi}{N}\right) .
$$

Hence we have

$$
\lim _{\varphi \rightarrow \pi / 2} \kappa(\varphi)=\pi-\frac{(m+1) \pi}{N} .
$$

We calculate $\kappa\left(\varphi_{\min }\right)$ by sending $\varphi$ to $\varphi_{\min }$ with $\varphi>\varphi_{\text {min }}$.

$$
\lim _{\varphi \rightarrow \varphi_{\text {min }}} \kappa(\varphi)=0 .
$$

We therefore have the following results. Case (vii): $x>0$ with complex deviation

$$
-\frac{m}{2} \leq J_{1}<\frac{N}{2}-\frac{1}{2}-m .
$$

Case (viii): $x<0$ with complex deviation

$$
-\frac{N}{2}+\frac{1}{2}<J_{1} \leq-\frac{m}{2} .
$$

Combining case (vii) and (viii) we have

$$
-\frac{N}{2}+\frac{1}{2}<J_{1}<\frac{N}{2}-\frac{1}{2}-m .
$$

Here we recall that $J_{2}$ is given by eq. (3.11): $J_{2}=J_{1}+m$ for $m=1,2, \ldots, N-1$. It is easy to show that the set of the Bethe quantum numbers $J_{1}$ and $J_{2}$ satisfying (3.42) corresponds to the set of $J_{1}$ and $J_{2}$ satisfying the following conditions:

$$
-\frac{N-2}{2}<J_{1}<J_{2}<\frac{N-2}{2} .
$$

They are nothing but the conditions of the quantum numbers for the standard 1string solutions with $M=2$ [10, 11]. Here we remark that when $N$ is even $J_{j}$ s are half-integers and hence $J_{j} \leq(N-2) / 2-1 / 2=(N-3) / 2$, which is equivalent to $J_{j}<(N-1) / 2$.

It is easy to show that the number of pairs $J_{1}$ and $J_{2}$ satisfying (3.11) and (3.42) is given by $(N-2)(N-3) / 2$, which coincides with the number of 1-strings in the $M=2$ sector expected by the string hypothesis. Thus, by deriving all the Bethe quantum numbers $J_{1}$ and $J_{2}$ exactly, we have shown the number of standard real solutions in the two down-spin sector. Moreover, it is consistent with the string hypothesis. 


\section{Some features of 2-string solutions}

\subsection{Singular string solution}

We now show that when $N$ is given by $N=4 n$ with an integer $n$, the Bethe quantum numbers $\left(J_{1}, J_{2}\right)=(N / 4-1 / 2, N / 4+1 / 2)$ and $(-N / 4-1 / 2,-N / 4+1 / 2)$, which correspond to the cases (i) and (ii), respectively, give the singular string solution $\left(\lambda_{1}, \lambda_{2}\right)=(i / 2,-i / 2)$. We also show that when $N$ is given by $N=4 n+2$ with an integer $n$, the Bethe quantum numbers $\left(J_{1}, J_{2}\right)=(N / 4, N / 4)$ and $(-N / 4,-N / 4)$, which correspond to the cases (iii) and (iv), respectively, give the singular string solution $\left(\lambda_{1}, \lambda_{2}\right)=(i / 2,-i / 2)$.

For $N=4 n$ we have $N / 4-1 / 2=n-1 / 2$, and it is a half-integer. Since $N$ and $M$ are even, the Bethe quantum numbers $J_{1}$ and $J_{2}$ are given by half-integers. Thus, $J_{1}=N / 4-1 / 2(\bmod 1)$, and hence $J_{1}$ can take the value $N / 4-1 / 2$. In the limit of sending the string deviation $\delta$ to 0 with $\delta>0$ we have

$$
\begin{aligned}
& \lim _{w \rightarrow 0 ; w>0} N Z(x(w), \delta(w)) \\
= & \lim _{w \rightarrow 0 ; w>0} \frac{N}{2 \pi}\left(\pi H(\delta)\left(\operatorname{sgn}(x)-\frac{1}{N}\right)+\tan ^{-1}\left(\frac{x}{1+\delta}(w)\right)-\tan ^{-1}\left(\frac{x}{\delta}(w)\right)\right) \\
= & \frac{N}{2 \pi}\left(\pi\left(1-\frac{1}{N}\right)+\tan ^{-1}(0)-\tan ^{-1}(\infty)\right) \\
= & \frac{N}{4}-\frac{1}{2} .
\end{aligned}
$$

Here we have made use of the small $w$-behavior:

$$
x /(1+\delta) \approx w^{1 / 2 N}(w \ll 1), \quad x / \delta \approx w^{1 / 2 N-1 / 2}(w \ll 1) .
$$

We have $J_{2}=J_{1}+1$ since $\delta>0$. We have $J_{2}=J_{1}+1=N / 4+1 / 2$. Thus, we have derived the singular solution by sending the string deviation $\delta$ to 0 with $\delta>0$. By setting $\epsilon=w^{1 / 2 N}$, we have

$$
\lambda_{1}=\epsilon+i / 2+i \epsilon^{N}, \quad \lambda_{2}=\epsilon-i / 2-i \epsilon^{N} .
$$

It is nothing but one of the regularization schemes for the singular string solution, where we send the positive small parameter $\epsilon$ to zero.

In the case of $\left(J_{1}, J_{2}\right)=(-N / 4-1 / 2,-N / 4+1 / 2)$, we consider case (ii) $\delta>0$ and $x<0$. We derive $J_{1}=-N / 4-1 / 2$ from the following limit:

$$
\begin{aligned}
& \lim _{w \rightarrow 0 ; w>0} \frac{N}{2 \pi}\left(\pi H(\delta)\left(\operatorname{sgn}(x)-\frac{1}{N}\right)+\tan ^{-1}\left(\frac{x}{1+\delta}(w)\right)-\tan ^{-1}\left(\frac{x}{\delta}(w)\right)\right) \\
= & \frac{N}{2 \pi}\left(\pi\left(-1-\frac{1}{N}\right)+\tan ^{-1}(0)-\tan ^{-1}(-\infty)\right) \\
= & -\frac{N}{4}-\frac{1}{2} .
\end{aligned}
$$

We thus obtain $J_{1}=-N / 4-1 / 2$. By setting $\epsilon=w^{1 / 2 N}$ we have

$$
\lambda_{1}=-\epsilon+i / 2+i \epsilon^{N}, \quad \lambda_{2}=-\epsilon-i / 2-i \epsilon^{N} .
$$

Here, the string center $x$ is negative: $x=-\epsilon$ and we send the small positive parameter $\epsilon$ to zero.

Similarly, we can show that for $N=4 n+2$ with an integer $n$, the Bethe quantum numbers $\left(J_{1}, J_{2}\right)=(N / 4, N / 4)$ and $(-N / 4,-N / 4)$ give the singular string solution: 
$\left(\lambda_{1}, \lambda_{2}\right)=(i / 2,-i / 2)$, by considering cases (iii) $\delta<0$ and $x>0$ and (iv) $\delta<0$ and $x<0$, respectively. In the case of $\left(J_{1}, J_{2}\right)=(N / 4, N / 4)$, by setting $\epsilon=w^{1 / 2 N}$ we have

$$
\lambda_{1}=\epsilon+i / 2-i \epsilon^{N}, \quad \lambda_{2}=\epsilon-i / 2+i \epsilon^{N} .
$$

Here, the string center $x$ is positive: $x=\epsilon$, while the string deviation $\delta$ is negative: $\delta=-\epsilon^{N}$, and we send the small positive parameter $\epsilon$ to zero.

In the case of $\left(J_{1}, J_{2}\right)=(-N / 4,-N / 4)$, by setting $\epsilon=w^{1 / 2 N}$ we have

$$
\lambda_{1}=-\epsilon+i / 2-i \epsilon^{N}, \quad \lambda_{2}=-\epsilon-i / 2+i \epsilon^{N} .
$$

Here, the string center $x$ is negative: $x=-\epsilon$, and the string deviation $\delta$ is negative: $\delta=-\epsilon^{N}$, and we send them to zero.

Here we remark that it has been observed through numerical solutions that the different quantum numbers correspond to the same singular string solution [35, 12. However, we have derived the four different sets of the Bethe quantum numbers corresponding to the same singular string solution, by solving the Bethe-ansatz equations in the logarithmic form with an analytic approach of sending the string deviation $\delta$ to zero. We recall that it is not possible to put $\delta=0$ directly in the BAEs. Hence, the singular string solution is different from the standard generic solutions of the BAEs. It satisfies the BAEs only in the limiting procedure.

\subsection{Large- $N$ behavior of 2-string solutions}

Let us consider case (i) when $\delta>0$ and $x>0$. We first recall (2.15). Sending $w$ to 1 we have

$$
\lim _{w \rightarrow 1} \frac{x}{\delta}=\sqrt{N-1} .
$$

When $w$ is close to 1 , both $x$ and $\delta$ are very large and we have $x \approx \sqrt{N-1} \delta$. Secondly, the largest Bethe quantum number $J_{1}$ satisfying (2.23) is given by $(N-3) / 2$. Putting $J_{1}=(N-3) / 2$ in to (2.7) we have

$$
-\frac{2 \pi}{N}=\tan ^{-1}(x /(1+\delta))-\tan ^{-1}(x / \delta) .
$$

Assuming that $x$ is large we apply the expansion: $\tan ^{-1}(x)=\pi / 2-1 / x+\cdots$, and we evaluate $x$ as $x \approx N / 2 \pi$. Thus, for $J_{1}=(N-3) / 2$, we have

$$
\delta \approx \frac{\sqrt{N}}{2 \pi} .
$$

\section{Completeness of the Bethe ansatz in the two down-spin sector}

\subsection{Enumeration of collapsed and non-collapsed 2-string solutions}

Let us now enumerate the number of solutions in the cases (i) to (vi). We combine the case (iii) with the case (v) and the case (iv) with the case (vi).

(A): $N=4 n$

We have one singular solution for either the case (i) or (ii), and $n-1$ generic solutions in each of the four cases, i.e., the cases (i), (ii), (iii) \& (v), and (iv) \& (vi). In total, we have $N-3$ solutions, since $4(n-1)+1=4 n-3=N-3$.

(B): $N=4 n+1$ 
We have no singular solution, and $n$ generic solutions in the cases (i) and (ii), $n-1$ generic solutions in the cases (iii) \& (v) and (iv) \& (vi). In total, we have $N-3$ solutions, since $2 n+2(n-1)=4 n-2=N-3$.

(C): $N=4 n+2$

We have one singular solution in either the case (iii) or (iv), $n$ generic solutions in the cases (i) and (ii), and $n-1$ generic solutions in the cases (iii) and (iv). In total, we have $N-3$ solutions, since $2 n+2(n-1)+1=4 n-1=N-3$.

(D): $N=4 n+3$

We have no singular solution, and $n$ generic solutions in each of the four cases. In total, we have $N-3$ solutions, since $2 n+2 n=4 n=N-3$.

Thus, we have shown that there are $(N-3) 2$-string solutions for any number of the lattice size $N$.

\subsection{Completeness through enumeration of the Bethe quantum numbers}

We have $(N-2)(N-3) / 2$ solutions for the 1-string and $N-3$ solutions for the 2 -string. In total we have

$$
\begin{aligned}
(N-2)(N-3) / 2+N-3 & =N(N-3) / 2 \\
& =N(N-1) / 2-N \\
& ={ }_{N} C_{2}-{ }_{N} C_{1}
\end{aligned}
$$

The number is consistent with that of the string hypothesis, which is given by the number of highest weight vectors under the total spin $S U(2)$ symmetry.

\subsection{Analytic derivation of the Takahashi quantum numbers}

Let us assume a 2 -string solution $\lambda_{1}=x+i(1+2 \delta) / 2$ and $\lambda_{2}=x-i(1+2 \delta) / 2$. We define the Takahashi quantum number $I$ for the solution by

$$
2 \tan ^{-1}\left(\frac{x}{1+\delta}\right)=\frac{2 \pi}{N} I
$$

We can show

$$
\lim _{w \rightarrow 0} 2 \tan ^{-1}\left(\frac{x(w)}{1+\delta(w)}\right)=2 \pi N\left(J_{1}+J_{2}\right)-\pi \operatorname{sgn}(x) .
$$

Therefore, we have the relation between the Takahashi quantum numebers $I$ and Bethe quantum numbers $J_{1}$ and $J_{2}$ as follows.

$$
I=J_{1}+J_{2}-\frac{N}{2} \operatorname{sgn}(x) .
$$

Let us now explain the derivation of the limit (5.3). For $2 \tan ^{-1} 2 \lambda_{1}$ we recall eq. (2.5). For $2 \tan ^{-1} 2 \lambda_{2}$ we have the following.

$$
\begin{aligned}
& 2 \tan ^{-1} 2 \lambda_{2}=2 \tan ^{-1}(2 x-i(1+2 \delta)) \\
& =\tan ^{-1}\left(\frac{x}{1+\delta}\right)-\tan ^{-1}\left(\frac{x}{\delta}\right) \\
& \quad+\pi H(\delta) \operatorname{sgn}\left(x_{+}\right)+\frac{1}{2 i} \log \left(\frac{x^{2}+\delta^{2}}{x^{2}+(1+\delta)^{2}}\right) .
\end{aligned}
$$


We therefore have the following:

$$
\begin{aligned}
& 2 \tan ^{-1} 2 \lambda_{1}+2 \tan ^{-2} 2 \lambda_{2} \\
& =2 \tan ^{-1}(2 x+i(1+2 \delta))+2 \tan ^{-1}(2 x-i(1+2 \delta)) \\
& =2 \tan ^{-1}\left(\frac{x}{1+\delta}\right)-2 \tan ^{-1}\left(\frac{x}{\delta}\right)+\pi H(\delta) \operatorname{sgn}\left(\left(x_{+}\right)+\operatorname{sgn}\left(x_{-}\right)\right)(5.6
\end{aligned}
$$

Here we remark that when $\delta$ is very small, then $w$ is also very small since we have eq. (2.15). When we take the limit of sending $w$ to zero, we have

$$
\frac{x}{\delta}=\epsilon_{2} \sqrt{\frac{w^{1 / N-1}-1}{1-w^{1 / N}}} \rightarrow \epsilon_{2} w^{1 / 2 N-1 / 2} \quad(w \rightarrow 0) .
$$

Hence we have

$$
2 \tan ^{-1}\left(\frac{x}{\delta}\right) \rightarrow 2 \tan ^{-1}\left(\epsilon_{2} w^{1 / 2 N-1 / 2}\right)=\epsilon_{2} \pi \quad(w \rightarrow 0) .
$$

Here we recall the relation: $\epsilon_{2}=\operatorname{sgn}(\delta) \operatorname{sgn}(x)$. By taking the sum of eqs. (2.2) and (2.3) we have

$$
2 \tan ^{-1} 2 \lambda_{1}+2 \tan ^{-2} 2 \lambda_{2}=\frac{2 \pi}{N}\left(J_{1}+J_{2}\right) .
$$

It follows from eq. (5.6). that the left-hand side of eq. (5.9) is given by

$$
\begin{aligned}
& 2 \tan ^{-1} 2 \lambda_{1}+2 \tan ^{-2} 2 \lambda_{2} \\
& =2 \tan ^{-1}\left(\frac{x}{1+\delta}\right)-2 \tan ^{-1}\left(\frac{x}{\delta}\right)+\pi H(\delta) \operatorname{sgn}\left(\left(x_{+}\right)+\operatorname{sgn}\left(x_{-}\right)\right) . \\
& =2 \tan ^{-1}\left(\frac{x}{1+\delta}\right)-\pi \operatorname{sgn}(\delta) \operatorname{sgn}(x)+\pi H(\delta)\left(\operatorname{sgn}\left(x_{+}\right)+\operatorname{sgn}\left(x_{-}\right)\right) \\
& =2 \tan ^{-1}\left(\frac{x}{1+\delta}\right)+\pi \operatorname{sgn}(x) .
\end{aligned}
$$

Here we note

$$
\begin{aligned}
& -\pi \operatorname{sgn}(\delta) \operatorname{sgn}(x)+\pi H(\delta)\left(\operatorname{sgn}\left(x_{+}\right)+\operatorname{sgn}\left(x_{-}\right)\right) \\
& =\left\{\begin{array}{cc}
-\pi \operatorname{sgn}(x)+2 \pi \operatorname{sgn}(x)=\pi \operatorname{sgn}(x) & \delta>0, \\
\pi \operatorname{sgn}(x) & \delta<0 .
\end{array}\right.
\end{aligned}
$$

Through the definition (5.2) we obtain the relation (5.4) between the Bethe quantum numbers $J_{1}$ and $J_{2}$ and the Takahashi quantum number $I$.

Considering the four cases: (A) $N=4 n$, (B) $N=4 n+1$, (C) $N=4 n+2$ and (D) $N=4 n+3$, we can show that the Takahashi numbers $I$ of the non-collapsed and collapsed 2-string solutions are distinct for all the $N-3$ solutions in any system size $N$. They are given by $-N / 2+2,-N / 2+3, \ldots, N / 2-2$ for any integer $N$.

\section{Discussion and concluding remarks}

\subsection{On the proof of the completeness}

In the paper we have derived the Bethe quantum numbers rigorously for all the solutions of the XXX spin chain in the two down-spin sector.

Since any complex solution with two down-spins is expressed in terms of two real parameters we have first solved BAE with respect to one of the two parameters and introduced the counting functions which give quantum numbers as their special values. 
We have shown that they are monotonic by explicitly calculating their derivatives and given analytically the upper and lower bounds to them. We have thus obtained the Bethe quantum numbers both for real and complex solutions in the two down-spin sector without making any assumption. We have thus shown that there are no more quantum numbers for physical solutions of BAE. We remark that even for real solutions we have sown that no other Bethe quantum numbers exist.

Moreover, we have shown that the derived set of the Bethe quantum numbers give exactly the same number to the dimensions of the vector space spanned by the eigenvectors of the XXX spin chain in the two down-spin sector. Therefore, if the Bethe-ansatz eigenvectors of the complex and real solutions associated with the derived set of the Bethe quantum numbers are linearly independent, then the derived Bethe-ansatz eigenvectors give a complete set of the subspace in the two down-spin sector.

It is shown by Slavnov that the inner product of two different Bethe-ansatz eigenvectors vanishes 40. Therefore, if we assume that the solutions of BAE for the derived set of the Bethe quantum numbers are distinct, then they lead to a complete set of the eigenvectors in the two down-spin sector. Although mathematically it has not been shown, yet, but it is quite likely that the solutions of BAE for the derived set of the Bethe quantum numbers are distinct, since the derived set consists of distinct pairs of integers or half-integers.

\subsection{Suggestions for further studies}

In the case of three down-spins the parametrization introduced in the paper is still useful and we can show some properties of the Bethe quantum numbers such as the difference of the quantum numbers associated with the string deviations. Some details will be given elsewhere.

Moreover, we can calculate the Bethe quantum numbers for the XXZ spin chain in the two down-spin sector by only slightly extending the method in the paper. For instance, we can derive the quantum numbers of 2-string solutions for the spin-1/2 XXZ chain in the gapful anti-ferromagnetic regime. We consider the XXZ Hamiltonian under the periodic boundary conditions:

$$
\mathcal{H}_{\mathrm{XXz}}=\frac{1}{2} \sum_{j=1}^{N}\left(\sigma_{j}^{x} \sigma_{j+1}^{x}+\sigma_{j}^{y} \sigma_{j+1}^{y}+\Delta \sigma_{j}^{z} \sigma_{j+1}^{z}\right),
$$

where $\sigma_{j}^{a}(a=x, y, z)$ are the Pauli matrices defined on the $j$ th lattice site. Let us express the XXZ anisotropy $\Delta$ by $\Delta=\cosh \zeta$ with $\zeta>0$. The Bethe-ansatz equations are given by

$$
\begin{aligned}
2 \tan ^{-1}\left(\frac{\tan \lambda_{j}}{\tanh (\zeta / 2)}\right)= & \frac{2 \pi}{N} J_{j}+\frac{1}{N} \sum_{k=1}^{M} 2 \tan ^{-1}\left(\frac{\tan \left(\lambda_{j}-\lambda_{k}\right)}{\tanh \zeta}\right) \\
& \text { for } \quad j=1,2, \ldots, M .
\end{aligned}
$$

Here the quantum numbers $J_{j}$ satisfy the condition: $J_{j}=(N-M+1) / 2(\bmod 1)$. We assume the folowing form of a 2-string:

$$
\lambda_{1}=x+\frac{i}{2} \zeta+i \delta, \quad \lambda_{2}=x-\frac{i}{2} \zeta-i \delta .
$$


We can express the string center $x$ in terms of deformation parameter $w=\tanh (\zeta / 2+$ $\delta) / \tanh (\zeta / 2)$ and $z=\tanh (\zeta / 2)$. Explicitly, the string center $x$ is given by

$$
\tan ^{2} x=\frac{1}{2 \mathcal{A}}\left(-\mathcal{B} \pm \sqrt{\mathcal{B}^{2}-4 \mathcal{A C}}\right)
$$

where $\mathcal{A}, \mathcal{B}$ and $\mathcal{C}$ are given by

$$
\begin{aligned}
\mathcal{A}= & w^{2}\left(1+w z^{2}\right)^{2}\left(\frac{1-w}{1+w} \frac{1-w z^{2}}{1+w z^{2}}\right)^{2 / N}-w^{2}\left(1-w z^{2}\right)^{2} \\
\mathcal{B}= & \left\{\left(1+w^{2} z^{2}\right)^{2} / z^{2}+2 w(1+w)\left(1+w z^{2}\right)\right\}\left(\frac{1-w}{1+w} \frac{1-w z^{2}}{1+w z^{2}}\right)^{2 / N} \\
& \quad-\left\{\left(1+w^{2} z^{2}\right)^{2} / z^{2}-2 w(1-w)\left(1-w z^{2}\right)\right\} \\
\mathcal{C}=(1+w)^{2} & \left(\frac{1-w}{1+w} \frac{1-w z^{2}}{1+w z^{2}}\right)^{2 / N}-(1-w)^{2} .
\end{aligned}
$$

We show that string deviation $\delta$ is negative with its absolute value being very small in order to make the string center $x$ being real-valued. Some more details will be discussed elsewhere.

\section{Appendix A. Formulas related to the logarithmic function}

For a given nonzero complex number $z=\alpha+i \beta$ where $\alpha$ and $\beta$ are real we express the $\log a r i t h m i c$ function $\log z$ by

$$
\log (\alpha+i \beta)=i(\theta(z)+2 \pi n)+\frac{1}{2} \log \left(\alpha^{2}+\beta^{2}\right),
$$

where $n$ is an integer $(n \in \boldsymbol{Z})$ corresponding to the branch of the logarithmic function and we express $\theta(z)$ as

$$
\theta(\alpha+i \beta)=\left\{\begin{array}{cc}
\tan ^{-1}(\beta / \alpha)+\pi H(-\alpha) \operatorname{sgn}\left(\beta_{+}\right) & \text {for } \alpha \neq 0 \\
\operatorname{sgn}\left(\beta_{+}\right) \pi / 2 & \text { for } \alpha=0
\end{array}\right.
$$

Here we recall that we take the branch: $-\pi / 2<\tan ^{-1} x<\pi / 2$ for $(x \in \boldsymbol{R})$. We denote by $\operatorname{sgn}\left(x_{+}\right)$a sign function $\operatorname{sgn}(x)$ shifted by an infinitesimally small positive number $0_{+}: \operatorname{sgn}\left(x_{+}\right)=\operatorname{sgn}\left(x+0_{+}\right)=1-2 H(-x)$.

The function $\theta(\alpha+i \beta)$ defined by $($ A.2 is continuous at $\alpha=0$ as a function of $\alpha$ when $\beta \neq 0$. The range is given by $-\pi<\theta(z)<\pi$ if $\beta \neq 0$, while if $\beta=0 \theta(z)=0$ or $\pi$ for $\alpha>0$ or $\alpha<0$, respectively.

We define the arctangent function $\tan ^{-1}(a+i b)$ for a nonzero complex number $a+i b$ where $a$ and $b$ are real by

$$
\tan ^{-1}(a+i b)=\frac{1}{2 i}(\log (1-b+i a)-\log (1+b-i a))
$$

Applying formula A.1 to A.3 we have for $b \neq \pm 1$

$$
\begin{aligned}
2 \tan ^{-1}(a+i b) & =\tan ^{-1}\left(\frac{a}{1-b}\right)+\pi H(b-1) \operatorname{sgn}\left(a_{+}\right) \\
& +\tan ^{-1}\left(\frac{a}{1+b}\right)+\pi H(-b-1) \operatorname{sgn}\left(a_{-}\right) \\
& +\frac{1}{2 i} \log \left(\frac{a^{2}+(b-1)^{2}}{a^{2}+(b+1)^{2}}\right) .
\end{aligned}
$$


In the branch: $-\pi<\operatorname{Im} \log z \leq \pi$ we can show

$$
\begin{array}{r}
-\pi<\operatorname{Re}\left(2 \tan ^{-1}(a+i b)\right)<\pi \quad(a \neq 0), \\
\operatorname{Re}\left(2 \tan ^{-1}(a+i b)\right)= \pm \pi \quad(a=0) .
\end{array}
$$

\section{Appendix B. Proof of lemma 2.1}

By dividing each side of inequality (2.22) by $\left(1+w^{1 / 2}\right)$ we reduce it to the following:

$$
\left(1-w^{1 / 2}\right)\left(1+w^{1 / N-1 / 2}\right)>N\left(1-w^{1 / N}\right) .
$$

Let us define $F(w)$ by $F(w)=\left(1-w^{1 / 2}\right)\left(1+w^{1 / N-1 / 2}\right)-N\left(1-w^{1 / N}\right)$. By taking the derivative we have

$$
F^{\prime}(w)=-\frac{w^{1 / N-3 / 2}}{2}\left(w^{1-1 / N}-2\left(1-\frac{1}{N}\right) w^{1 / 2}+1-\frac{2}{N}\right) .
$$

We define $G(w)$ by $G(w)=w^{1-1 / N}-2(1-1 / N) w^{1 / 2}+1-2 / N$. The derivative of $G(w)$ is given by

$$
G^{\prime}(w)=(1-1 / N) w^{-1 / 2}\left(w^{1 / 2-1 / N}-1\right)
$$

It is clear that $G^{\prime}(w)<0$ for $0<w<1$. Since $G(1)=0$, we have $G(w)>0$ for $0<w<1$. Therefore we have $F^{\prime}(w)<0$ for $0<w<1$. Since $F(1)=0$, we have $F(w)>0$ for $0<w<1$. Hence, we obtain inequality (2.22).

\section{Appendix C. Proof of inequality (3.20)}

Multiplying both hands side of (3.20) by $\sin ^{2}(2 \varphi / N) \cos ^{5} \varphi / \sin \varphi$ we show that (3.20) is equivalent to

$$
\cos ^{2}\left(\varphi-\frac{2 \varphi}{N}\right)-\cos \varphi \cos \left(\varphi-\frac{2 \varphi}{N}\right) \frac{\sin 2 \varphi}{N \sin (2 \varphi / N)}>\sin \left(\frac{2 \varphi}{N}\right) \sin \left(2 \varphi-\frac{2 \varphi}{N}\right) \cdot(\mathrm{C} .
$$

Making use of $\sin \alpha \sin \beta=(\cos (\alpha-\beta)-\cos (\alpha+\beta)) / 2$ and $\cos ^{2} \alpha=(1+\cos 2 \alpha) / 2$ in the right-hand side and left-hand side, respectively, and multiplying both hand sides by $\sin (2 \varphi / N) / \cos ^{2} \varphi$ we reduce it to

$$
\sin \left(\frac{2 \varphi}{N}\right)>\frac{2}{N} \sin \varphi \cos \left(\varphi-\frac{2 \varphi}{N}\right) \quad 0<\varphi<\pi / 2 .
$$

By applying (3.16) in lemma 3.1 with $\alpha=2 / N$ and making use of the fact that $1>\cos \varphi-2 \varphi / N)>0$ we show the reduced inequality (C.2).

\section{Appendix D. Proof of inequality (3.35)}

Multiplying both hands side of (3.20) by $\sin ^{2}((2 \varphi+m \pi) / N) \cos ^{5} \varphi / \sin \varphi$ we show that (3.35) is equivalent to

$$
\begin{aligned}
& \cos ^{2}\left(\varphi-\frac{2 \varphi+m \pi}{N}\right)-\cos \varphi \cos \left(\varphi-\frac{2 \varphi+m \pi}{N}\right) \frac{\sin 2 \varphi}{N \sin ((2 \varphi+m \pi) / N)} \\
& >\sin \left(\frac{2 \varphi+m \pi}{N}\right) \sin \left(2 \varphi-\frac{2 \varphi+m \pi}{N}\right) \text { for } \frac{m \pi}{2(N-1)}<\varphi<\frac{\pi}{2} .
\end{aligned}
$$


Making use of $\sin \alpha \sin \beta=(\cos (\alpha-\beta)-\cos (\alpha+\beta)) / 2$ and $\cos ^{2} \alpha=(1+\cos 2 \alpha) / 2$ in the right-hand side and left-hand side, respectively, and multiplying both hand sides by $\sin ((2 \varphi+m \pi) / N) / \cos ^{2} \varphi$ we reduce it to

$$
\begin{gathered}
\sin \left(\frac{2 \varphi+m \pi}{N}\right)>\frac{2}{N} \sin \varphi \cos \left(\varphi-\frac{2 \varphi+m \pi}{N}\right) \\
\text { for } \frac{m \pi}{2(N-1)}<\varphi<\frac{\pi}{2} .
\end{gathered}
$$

Here we show the following inequality:

$$
\begin{gathered}
\sin \left(\frac{2 \varphi+m \pi}{N}\right)>\frac{2}{N} \sin \varphi \cos \left(\varphi-\frac{2 \varphi+m \pi}{N}\right) \\
\text { for } 0<\varphi<\pi .
\end{gathered}
$$

We define function $f(\varphi)$ by

$$
f(\varphi)=\sin \left(\frac{2 \varphi+m \pi}{N}\right)-\frac{2}{N} \sin \varphi \cos \left(\varphi-\frac{2 \varphi+m \pi}{N}\right)
$$

By taking the derivative we have

$$
\frac{d f}{d \varphi}=\frac{4}{N}\left(1-\frac{1}{N}\right) \sin \varphi \sin \left(\varphi-\frac{2 \varphi+m \pi}{N}\right) .
$$

The derivative vanishes at $\varphi=m \pi /(N-2)$, and hence function $f(\varphi)$ has the lowest value at $\varphi=m \pi /(N-2)$. By putting $\cdot \varphi=m \pi /(N-2)$ we have

$$
f\left(\varphi_{\min }\right)=\left(1-\frac{2}{N}\right) \sin \left(\frac{m \pi}{N-2}\right) .
$$

The right-hand side is positive for $m=1,2, \ldots, N-2$ and vanishes for $m=0$ and $N-1$. We therefore obtain the following:

$$
\sin \left(\frac{2 \varphi+m \pi}{N}\right)>\frac{2}{N} \sin \varphi \cos \left(\varphi-\frac{2 \varphi+m \pi}{N}\right) \text { for } 0<\varphi<\pi .(\mathrm{D} .7)
$$

\section{Acknowledgements}

The authors would like to thank Profs. J.-S. Caux and A. N. Kirillov for useful comments. The present study is partially supported by Grant-in-Aid for Scientific Research No. 15K05204. Some part of the present research is performed when participating in the YITP workshop: New Frontiers in Non-equilibrium Physics 2015, July 21st - August 23rd, 2015, YITP, Kyoto University.

[1] H. A. Bethe, Z. Phys. 71 205-226 (1931).

[2] M. Gaudin, "La Fonction d' Onde de Bethe", (Paris: Masson) (1983).

[3] R. Baxter, Exactly Solved Models in Statistical Mechanics, (Academic Press, New York, 1982).

[4] L. D. Faddeev and L. A. Takhtajan, J. Sov. Math. 24241 (1984).

[5] E. K. Sklyanin, J. Soviet Math. 31 (1985) 3417-3431; E. K. Sklyanin, The quantum Toda chain, in Non-linear equations in classical and quantum field theory, ed. N. Sanchez, (Springer, 1985). (Lect. Notes in Physics 226 (1985) 196-233.)

[6] V. Korepin, N. Bogoliubov and A. Izergin, "Quantum Inverse Scattering Method and Correlation Functions", (Cambridge: Cambridge University Press) (1993).

[7] L. D. Faddeev, Les Houches Summer School Proceedings, 64, Ed. A. Connes, K. Gawedzki and J. Zinn-Justin (Amsterdam: North Holland) 149-219 (1995), (arXiv: hep-th/9605187). 
[8] B. Sutherland, "Beautiful Models: 70 Years of Exactly Solved Quantum Many-Body Problems", (Singapore: World Scientific) (2004).

[9] T. Koma and H. Ezawa, Prog. Tehor. Phys. 781009 (1987).

[10] M. Takahashi, Prog. Theor. Phys. 46401 (1971).

[11] M. Takahashi, "Thermodynamics of One-Dimensional Solvable Models", (Cambridge: Cambridge University Press) (1999).

[12] R. Hagemans and J.-S. Caux, J. Phys. A: Math. Theor. 40 14605-14647 (2007).

[13] W. Hao, R. I. Nepomechie and A. J. Sommese, Phy. Rev. E 88052113 (2013).

[14] P. R. Giri, T. Deguchi, J. Stat. Mech., P07007 (2015).

[15] A. N. Kirillov and R. Sakamoto, J. Phys. A. Math. Theor. 47205207 (2014).

[16] F. H. L. Essler, V. E. Korepin and K. Schoutens, J. Phys. A: Math. Gen. 254115 (1992).

[17] A. A. Vladimirov, Theor. Math. Phys. 66, 102 (1986).

[18] J.-S. Caux, private communication, July, 2015.

[19] A. N. Kirillov and N. Yu. Reshetikhin, J. Sov. Math. 41 925-955 (1988).

[20] A. N. Kirillov, J. Geom. Phys. 5 365-389 (1988).

[21] A. N. Kirillov, St. Petersburg Math. J. 12 161-190 (2001).

[22] B. Lulek, T. Lulek, M. Labuz and R. Stagraczynski, Physica B 405 2654-2658 (2010).

[23] L. V. Avdeev and A. A. Vladimirov, Theor. Math. Phys. 69, 1071 (1987).

[24] R. Siddharthan, arXiv:cond-mat/9804210

[25] N. Beisert, J. A. Minahan, M. Staudacher and K. Zarembo, JHEP 09010 (2003).

[26] R. I. Nepomechie and C. Wang, J. Phys. A: Math. Theor. 46325002 (2013).

[27] R. I. Nepomechie and C. Wang, J. Phys. A: Math. Theor 47505004 (2014).

[28] W. Hao, R. I. Nepomechie and A. J. Sommese, J. Stat. Mech. P03024 (2014).

[29] A. N. Kirillov and R. Sakamoto, arXiv: 1406.1958v3 [math-ph].

[30] T. Deguchi, P. R. Giri, J. Stat. Mech., P02004 (2015).

[31] P. R. Giri, T. Deguchi, J. Phys. A: Theor. Math. 48, 175207 (2015).

[32] A. A. Vladimirov, Phys. Lett. A 105 418-420 (1984).

[33] K. Isler and M. B. Paranjape, Phys. Lett. B 319209 (1993).

[34] A. Ilakovac, M. Kolanović, S. Pallua and P. Prester, Phys. Rev. B 607271 (1999).

[35] T. Fujita, T. Kobayashi and H. Takahashi, J. Phys. A: Math. Gen. 36 1553-1564 (2003).

[36] F. Woynarovich, J. Phys. A 152985 (1982).

[37] O. Babelon, H. J. de Vega and C. M. Viallet, Nucl. Phys. B 220 13-34 (1983).

[38] K. Fabricius and B. M. McCoy, J. Stat. Phys. 103 647-678 (2001).

[39] K. Fabricius and B. M. McCoy, J. Stat. Phys. 104 573-587 (2001).

[40] N. A. Slavnov, Russian Math. Surveys 62, 727-766 (2007). 